\title{
PARLER DE FOUTRE : LA DÉSIGNATION DES PARTIES DU CORPS DANS LES FABLIAUX MÉDIÉVAUX
}

Ondřej PEŠEK

Université de Bohême du Sud, České Budějovice

\begin{abstract}
En): The aim of the study is to analyze the processes of naming the body parts in medieval French fabliaux. The analysis is based on a lexicometric research carried out by the TXM software; the corpus of the fabliaux having been digitalized. The data obtained are interpreted in relation to the philological specificities of the analyzed corpus.
\end{abstract}

Keywords (En): body parts; fabliaux; lexicometry; old French

Mots-clés (Fr) : parties du corps ; fabliaux ; lexicométrie ; ancien français

DOI : $10.32725 /$ eer.2021.009

\section{Introduction}

Dans la présente étude, nous analyserons les procédés de dénomination des parties du corps dans les fabliaux médiévaux. L'analyse est basée sur la lexicométrie effectuée à l'aide du logiciel TXM sur un corpus numérisé des fabliaux. Les données ainsi obtenues seront interprétées relativement aux spécificités philologiques du corpus analysé.

\section{Fabliaux médiévaux - thématiques privilégiées et fonctions principales}

Le nom de fabliaux désigne un ensemble de contes comiques et frivoles, composés en vers que l'on voit naître dans la littérature de la langue d'oïl de la fin du XII ${ }^{e}$ siècle au premier tiers du XIV ${ }^{e}$ siècle. Le nombre de textes que l'on range sous cette appellation ne fait pas l'unanimité, les critères formels, thématiques et/ou compositionnels étant trop flous pour pouvoir circonscrire un corpus clos et clairement délimité ${ }^{1}$. Plutôt que d'énumérer les propriétés définitoires qui auraient l'ambition d'embrasser intégralement les fabliaux dans leur diversité et de postuler des dénominateurs communs, si sophistiqués soient-ils ${ }^{2}$, nous préférons souligner quelques traits prototypiques qui, sans être forcément partagés par tous les textes fabliesques, en présentent les caractéristiques les plus saillantes. Parmi celles-ci, il faut citer en premier lieu les aventures érotiques, qui représentent la thématique prototypique des fabliaux. Comme les fabliaux ont été écrits pour faire rire, ils amplifient tous les aspects comiques et ridicules qui naissent dans et autour des

\footnotetext{
${ }^{1}$ La question relative au "nombre » de fabliaux à retenir dans la littérature française est traitée en détail par A. CORBELLARI, 2014.

${ }^{2}$ La vanité d'une telle entreprise a été pertinemment pointée par P. MÉNARD (1983).

${ }^{3}$ Et c'est peut-être le seul élément définitoire partagé par tous les textes : $c f$. le sous-titre que donne P. Ménard à son ouvrage de 1983 - «Contes à rire du Moyen Âge ».
} 
rapports sexuels entre l'homme et la femme ${ }^{4}$. Moines et prêtres lubriques, femmes insatiables, maris trompés, jeunes hommes performants sont autant de protagonistes typiques des intrigues amoureuses. Leurs aventures et mésaventures sont racontées sans ambages, car dans le domaine de la sexualité, l'explicite sert directement les effets humoristiques, qui représentent, comme on l'a vu, la première fonction des fabliaux. Comme le dit Michel ZINK dans son Avertissement (1993: 8) : «à la prétérition chère à la littérature courtoise, à son art du détour pour dire le désir et à son refus d'une expression directe de l'innommable, répond dans les fabliaux, de façon inversée et symétrique, un gros plan exclusif et permanent, à vrai dire assez peu érotique à notre goût, sur les parties génitales et sur l'acte sexuels. » Dans les textes, on relève ainsi des dénominations directes, dont l'obscénité représente une marque distinctive du genre fabliesque, mais à côté de ces expressions « franches », on trouve aussi des tournures imagées qui témoignent du fait que le seul acte de nommer les organes sexuels entraîne un sentiment d'embarras dont la résolution a tout le potentiel de faire rire. Aussi l'analyse de la langue des fabliaux permet-elle de documenter les pratiques discursives servant à nommer les parties du corps pudiques, tout en tenant compte de la fonction comique que ces dénominations sont censées remplir conformément au contrat du genre.

\section{Enquête lexicographique préliminaire}

Avant de présenter la méthodologie et les résultats de notre étude, nous voulons faire quelques remarques générales qui justifient les hypothèses sur lesquelles notre analyse est basée. Ces remarques résultent d'une enquête lexicographique qui a précédé notre analyse de corpus de la langue des fabliaux. Cette enquête, menée à titre complémentaire, a été basée sur les données lexicales que fournit le Dictionnaire $d u$ Moyen Français (DMF) disponible en ligne (http://atilf.atilf.fr/dmf/). Grâce aux fonctionnalités que contient cet excellent dictionnaire, nous avons recensé les entrées qui désignent les parties du corps humain ${ }^{5}$. Nous avons constaté trois faits majeurs :

1) Du point de vue quantitatif, les expressions qui désignent les organes génitaux masculins et féminins (testicules, sexe masculin, sexe féminin) prédominent d'une manière particulièrement significative (ensemble, elles représentent $45 \%$ de toutes les expressions retenues).

2) Il n'est pas facile d'identifier un élément «neutre » qui, à l'instar du mot «jambe» par exemple, pourrait fonctionner en français médiéval comme

\footnotetext{
${ }^{4}$ Conformément aux attitudes médiévales, les rapports homosexuels sont absents des textes des fabliaux. Quant aux pratiques sexuelles elles-mêmes, que les fabliaux exhibent à volonté, elles restent somme toute assez «ordinaires » : sodomie, fellation, masochisme, sadisme, inceste n'ont aucune place dans la description du coït ( $c f$. R. H. BLOCH, 1993 : 536).

${ }^{5}$ Fondant notre enquête sur les éléments lexicaux « de base » que contient la définition des entrées, nous avons recherché les dénominations relatives aux parties du corps suivantes (le chiffre entre parenthèses correspond au nombre de dénominations différentes identifiées respectivement pour chacune des parties du corps) : bouche (6), nez (6), oreille (3), xil (1), visage (17), gorge (25), poitrine (8), sein (4), mamelle (6), ventre (25), bras (5), jambe (7), dos (3), tête (11), main (7), cour (4), fesses (11), testicule (22), sexe masculin (62), sexe féminin (41).
} 
dénomination des parties pudiques à travers tous les styles, genres ou types de discours.

3) La plupart des expressions qui désignent les parties génitales sont de nature métaphorique ${ }^{6}$.

Des éléments d'explication de ces constats sont à chercher au niveau culturel. On sait bien que dans la culture occidentale, les organes sexuels sont cachés par les vêtements et toute découverte volontaire peut être considérée comme une atteinte à la morale et à l'ordre public. Cette pratique culturelle est reflétée par la langue parler de ces organes est toujours une affaire délicate. Mais comme la procréation et l'acte sexuel qui y est inséparablement lié représentent le désir central de l'humanité, les locuteurs éprouvent naturellement un grand besoin d'en parler. D'où un paradoxe apparent : les organes dont il n'est pas bienséant de parler en public font l'objet d'une activité onomasiologique extraordinaire. La question sexuelle étant sulfureuse par définition, les fonctions ludiques et cryptiques se complètent pour motiver la créativité lexicale constatée. Pour «cacher», on évite des dénominations directes, pour « amuser », les dénominations indirectes que l'on crée sont toujours marquées par un petit brin d'humour.

À l'issue de cette enquête préliminaire, nous avons formulé trois questions de recherche qui permettent d'orienter notre analyse de corpus. Les voici en résumé :

1) La prépondérance quantitative des expressions désignant les parties du corps pudiques est frappante et suscite nécessairement des questions relatives à la stabilité des éléments qui fonctionnent dans un texte comme dénominations des organes corporels (typiquement sexuels) - ceci concerne plus particulièrement la « lexicalisation » des métaphores.

2) Le statut diastratique des expressions désignant les parties du corps est difficile à déterminer : y a-t-il des indices qui permettent de statuer à ce propos ?

3) Le discours relatif aux organes sexuels reflète-t-il le statut particulier que notre culture (ou bien la culture occidentale dans sa dimension historique) réserve à ces parties du corps?

Ces questions-ci seront abordées au fil des analyses suivantes qui, sans aspirer à donner une réponse tranchée et définitive, se veulent une contribution modeste à cette problématique complexe.

\section{Le corpus des fabliaux}

Les fabliaux, comme nous l'avons vu, sont principalement des contes à rire. Leur valeur esthétique, leur place dans l'histoire de la littérature française, leur composition et leur poétique ont été décrites et analysées en détail : la littérature

\footnotetext{
${ }^{6}$ En voici quelques exemples. Le sexe masculin : aiguille, andouille, boyau, bras, corne, chandelle, perche, queue, verge, rable (ressemblance formelle); arc, contrelance, dague, lance, saqueboute, brant, perchant (armes); asperge, martelet, escourgeon, bourdon, escorie, fuseau, cranequin, engin, loque, clef (outils). Les testicules (ressemblance formelle et fonctionnelle): boulettes, cloches, clochettes, dandrilles, maillets, martelets, prunelles, doucettes. Le sexe féminin (ressemblance formelle et fonctionnelle) : cage, coffre, conduit, plaie, sac, gaine, creuset, cabat, baquet, cran, hostel, enclume, ouvroir.
} 
spécialisée en la matière est particulièrement abondante ${ }^{7}$. Une étude lexicale des fabliaux, philologique par définition, doit en tenir compte rigoureusement. Étant donné toutefois que ces aspects, aussi déterminants soient-ils pour les choix lexicaux opérés, ne font pas l'objet premier de notre étude, nous n'allons pas les relater ici et nous renvoyons le lecteur à la littérature secondaire. Conscients de la complexité philologique de la matière, nous entrons directement dans le vif du sujet en présentant les résultats de nos analyses de corpus.

Précisons tout d'abord les propriétés techniques de notre corpus de fabliaux. En ce qui concerne le contenu, il est basé sur l'édition MONTAIGLON-RAYNAUD (désormais M-R, cf. Bibliographie). Nous sommes conscients que cette édition, jugée avec presque 150 ans de recul, est loin d'être parfaite tant du point de vue qualitatif (les pratiques éditoriales ayant considérablement évolué) que quantitatif ${ }^{8}$. Cependant, elle a, à nos yeux, deux atouts majeurs : d'abord, elle est la plus compréhensive par rapport aux autres, mais surtout, elle est disponible au format pdf $^{9}$. Facilement numérisable grâce aux logiciels OCR, cette édition a pu servir de base pour la constitution d'un corpus électronique explorable par des outils computationnels modernes. Ce travail exigeant et minutieux a été effectué par Petr Březina et Pavla Konrádová, étudiants à la Faculté des Lettres de České Budějovice, qui ont préparé, sur la base des fiches obtenues à partir de l'édition $\mathrm{M}-\mathrm{R}$, la version électronique des textes ${ }^{10}$. Ces textes ont ensuite été téléchargés en tant que corpus complet $^{11}$ dans le logiciel $\mathrm{TXM}^{12}$ qui permet, grâce à ses fonctionnalités très développées, d'effectuer des analyses lexicométriques avancées. Le corpus est annoté par le système TreeTagger (ancien français - fro) qui parvient, d'une manière assez fiable, à étiqueter les parties du discours ${ }^{13}$.

La première étape de notre recherche de corpus consiste en une analyse statistique du lexique. Nous sommes conscients des limites des méthodes quantitatives ${ }^{14}$, ainsi, les conclusions que nous tirons sur la base de ces données brutes sont formulées avec peu d'ambition et beaucoup de précautions. Malgré leurs limites les analyses computationnelles statistiques présentent des avantages considérables, nous en citons quatre qui nous paraissent les plus significatives :

1) constituées sur la base du seul critère quantitatif, les listes lexicales obtenues sont objectives ;

2) les calculs effectués par l'ordinateur sont précis, rapides et exhaustifs ;

3) les analyses statistiques computationnelles sont facilement reproductibles, ce qui a trait à leur fiabilité ;

4) les fonctionnalités des logiciels de corpus permettent une navigation rapide et ordonnée à travers des entités textuelles de grande envergure.

\footnotetext{
${ }^{7}$ Cf. en particulier J. Bedier (1893), P. NyKrog (1973), P. Menard (1983), D. Boutet (1985).

${ }^{8}$ Cf. en particulier A. CORBELlari (2014).

${ }^{9}$ Cf. https://gallica.bnf.fr/accueil/fr/content/accueil-fr?mode=desktop

${ }^{10} \mathrm{Je}$ tiens à les remercier ici, car sans leur travail précieux, cette étude ne pourrait pas voir le jour.

${ }^{11}$ Les fichiers ont été convertis au format xml ; chaque fabliau correspond à un fichier, qui devient « texte » dans le corpus TXM.

${ }^{12} \mathrm{Cf}$. http://textometrie.ens-lyon.fr/

${ }^{13}$ Le système ne permet pas, pour l'instant, de lemmatiser les textes. Cette limitation ne représente pas pour autant un obstacle majeur pour les analyses.

${ }^{14}$ Cf. O. PEŠEK (2019).
} 
Cependant, quelles que soient la rapidité, la précision et l'envergure des calculs computationnels, ils ne fournissent, au bout du compte, que des indices, qui nécessitent une interprétation ultérieure faite par un chercheur conformément aux buts de son analyse.

\section{Spécificités lexicales générales du corpus des fabliaux}

Pour pouvoir évaluer les spécificités lexicales du corpus des fabliaux (corpus Fabliaux), il est nécessaire de faire contraster les données statistiques obtenues avec les profils lexicaux d'autres corpus textuels, constitués d'une manière pertinente du point de vue philologique ${ }^{15}$. Aussi avons-nous comparé les statistiques lexicales du corpus Fabliaux à celles du corpus constitué de romans en vers rédigés entre 1150 et 1340 (corpus Romans). Le corpus a été établi dans le cadre de la Base du français médiéval ${ }^{16}$, nous avons appliqué les critères de sélection suivants : forme - [vers] ; date œuvre - [1150-1340]; genre - [roman]. Des textes obtenus ainsi par une sélection automatique nous avons exclu manuellement le Roman de la Rose de Jean de Meun, qui représente, du point de vue générique et thématique, un cas à part par rapport aux autres éléments retenus. Le corpus contient ainsi des romans « courtois» et des romans "antiques». Du point de vue philologique, il est suffisamment contrastif: sur une base commune (textes composés en vers, provenant de la même époque), il représente un échantillon différent quant aux propriétés génériques. Voici les caractéristiques sommaires des deux corpus :

\begin{tabular}{|l|l|l|}
\hline Entité & Corpus Fabliaux & Corpus Romans \\
\hline Nombre de mots & 308539 & 707410 \\
\hline Nombre de textes & 157 & 19 \\
\hline \multicolumn{2}{|r}{ Tableau 1: Caractéristiques quantitatives des corpus }
\end{tabular}

Après avoir établi les deux corpus, nous en avons calculé les fréquences lexicales. Étant donné la thématique de ce volume (parties du corps), nous avons limité notre recherche aux seuls substantifs ; comme le corpus Fabliaux n'est pas lemmatisé, les données obtenues sont des entités «word». Afin de garder les mêmes paramètres de recherche, l'enquête menée dans le corpus Romans a été réglée avec les mêmes paramètres ${ }^{17}$. Comme les tailles des deux corpus ne sont pas strictement les mêmes, nous avons converti les fréquences absolues en fréquences relatives, calculées en nombre d'items par million. Ces données statistiques permettent de comparer la distribution des fréquences lexicales dans les deux corpus. Ainsi avons-nous comparé les occurrences d'items lexicaux qui présentent

${ }^{15}$ Cf. en particulier, F. RASTIER (2011).

16 Dans son corpus principal (BFM2019), la Base de Français Médiéval comporte 170 textes (4 700000 mots) écrits entre le IX et la fin du XV siècle. $C f$. http://bfm.ens-lyon.fr/

${ }^{17}$ Bien que la BFM permette d'enquêter sur les lemmes. 
une fréquence relative supérieure ou égale à 100 i.p.m ${ }^{18}$. Les résultats de cette enquête ne sont pas surprenants, et confirment ce que nous savons des thématiques traitées respectivement dans les deux ensembles génériques contrastés. Mais comme nous l'avons souligné plus haut : l'enquête computationnelle donne, relativement aux spécificités des profils lexicaux des textes, une preuve exacte et contrôlable. Ainsi, le corpus Romans contient 228 mots-formes qui correspondent au seuil fréquentiel donné, le corpus Fabliaux, lui, en contient 210. Sur ces ensembles, 145 mots-formes figurent dans les deux listes, soit 103 lemmes (lemmatisé manuellement). Il s'agit, pour la plupart, des mots les plus courants (jours, nuit, pere, mere, sire, dame, vaslez, vie, mort, terre, hons, chose, chevaus, ami, vin etc.). Quant aux parties du corps, la liste commune comporte les expressions suivantes: chief, teste, braz, cuer, col, piez, main. Mais naturellement, ce sont les mots-formes contenus sur l'une des deux listes seulement qui nous informent sur les spécificités lexicales du corpus. Ainsi, dans le corpus Fabliaux (car c'est celui qui nous intéresse plus particulièrement), nous comptons 52 lemmes différentiels. Parmi eux, les éléments suivants sont les plus caractéristiques : borgois, mari, meschine, prestre, moines, clers, vilain, vit, cul, con, coille. Ainsi, le calcul computationnel résume parfaitement l'enjeu des textes du corpus - parler du foutre pour faire rire le public.

\section{Les dénominations des parties du corps pudiques}

Comme nous venons de le voir, l'enquête statistique a relevé l'importance quantitative des noms des organes pudiques (vit, cul, con, coille) dans les fabliaux. La fréquence élevée de ces mots, qui représentent des dénominations directes de ces organes, est même l'une des marques distinctives du genre - leur fréquence dans le corpus Fabliaux est d'autant plus significative que dans le corpus Romans, ces expressions ne comptent pas une seule occurrence. Conformément aux objectifs de cette étude, nous allons analyser en détail la nature et la valeur de ces mots, telles qu'elles se présentent à travers les textes médiévaux.

\subsection{Valeur diastratique des dénominations directes}

De ces quatre expressions, l'élément le plus fréquent est le mot vit (rang 42), viennent ensuite cul (rang 95), con (rang 112) et coille (rang 168). Nous avons souligné plus haut (section 3) les difficultés qu'on a à déterminer la valeur diastratique de ces expressions en français médiéval. Dans les lignes qui suivent, nous essaierons de donner quelques éléments de réponse à cette question complexe. Pour ce faire, nous analyserons trois textes médiévaux qui, ayant pour objet la matière lexicale relative aux parties du corps pudiques, comportent des commentaires métalinguistiques ou des propositions terminologiques explicites. Le premier de ces textes est le Roman de la Rose de Jean de Meun; il contient de nombreux passages qui dissertent longuement sur la valeur des mots. Nous commenterons ici les extraits les plus significatifs pour notre propos. Ainsi, dans les

\footnotetext{
${ }^{18}$ Cette comparaison a été faite manuellement, sur la base des listes de mots exportées sous format
} xlsx. 
vers (vv. 6898-6901), l'Amant fait un reproche à la Raison: Si ne vos tiegn pas a cortaise / quant ci m'avez coilles nomees, / qui ne sunt pas bien renomees / en bouche a cortaise pucele... ${ }^{19}$ La Raison lui répond que le mot n'est pas en cause, car il ne fait qu'appeler les «bonnes choses » créées par Dieu. Le mot lui-même est, selon la Raison, «beau »: Coilles est biaus nons et si l'ains, / si sunt par foi coillon et vit, onc nus plus biaus guieres ne vit (vv. 7084-7086). Conment, por le cors seint Homer ! / n'oseré je mie nomer / proprement les euvres mon pere? (7094 -7096). Et la Raison de continuer: Se fames nes noment en France, ce n'est fors desacoutumance, car li propres nons leur pleüst, qui acoutumé leur eüst ; (vv. 71017104). Ainsi les mots coilles et vit sont les mots donnés «proprement » aux choses qui, créées par Dieu, ne peuvent avoir en soi rien de mauvais. Il s'agit d'expressions «normales » («propres ») et si les femmes ne les utilisent pas, c'est parce qu'elles n'y sont pas habituées. Selon la Raison, c'est plutôt la matière qui suscite un sentiment de pudeur et non pas les mots mêmes. L'usage de l'expression n'est qu'une question d'habitude ${ }^{20}$. À moins que Jean de Meun ne recherche un effet comique au deuxième plan, tout porte à croire que la langue française de l'époque n'avait pas à sa disposition un autre mot d'usage courant pour désigner les parties en question.

Le deuxième texte que nous avons analysé en vue de notre propos vient corroborer cette thèse. Il s'agit de l'extrait du traité Placides et Timeo, consacré aux organes génitaux de l'homme et de la femme. Traité scientifique et didactique par excellence $^{21}$, le langage de ce texte se veut savant et refuse tout élément de connotation malséante. Dans l'extrait analysé, l'auteur parle des organes génitaux dans les termes suivants : Nous apelons le malle celui qui a deux genitaires [...] qui sont appelees "testiculi » en latin et c'est a dire petis tesmoings du sexe a l'omme pour savoir se il est malles ou fumelle. Et si couvient que li homs ait une vergue qui soit chef de toutes les vaines de dessus soy, si est apelee "preapus ", qui est dit de "primo », qui vaut a dire premierement, et de apparans » [...] la quelle vergue est apelee "preape » et en commune langue «vit» (100-101). Les organes sexuels de la femme sont décrits d'une manière plus complexe (113-114). L'auteur parle «d'orefices»-Nous apelons orefice, qui est en latin de "os» qui vaut à dire " bouque ». Pour ce qui est des organes de la reproduction, l'anatomie en est, selon l'auteur, la suivante :... le secont orefice si est apelés "vulva», car il est dis selon aucuns philosophes de "volvens", ce est a dire tournans, car icelle vulve si tourne [...] et pour ce est chus orefices apelés vulve. Et ce boel a quoy tient celle vulve si est tout ensemble apelés marris, de matrix en latin, et chus " matrix » si est dit de

${ }^{19}$ Conformément au projet de Jean de Meun, ce passage fait écho aux vers 2109-2114 de Guillaume de Lorris, auteur de la première partie du Roman de la Rose, dans lesquels l'Amour incite l'Amant à ne pas prononcer des mots « infâmes »: Aprés gardes que tu ne dies / ces orz moz ne ces ribaudies... Je ne tieng pas a courtois ome qui orde chose e laide nome.

${ }^{20}$ Comme le note A. Strubel dans son édition du Roman de la Rose (p. 393, 395), la Raison défend ici une position nominaliste : le mot est attribué à la chose de manière arbitraire. Cette position amène la Raison à dénoncer toute hypocrisie linguistique dont la courtoisie n'est que trop affectée.

${ }^{21}$ L'œuvre Placides et Timéo ou Li secrés as philosophes est un traité didactique anonyme rédigé dans la deuxième moitié du XIII ${ }^{\mathrm{e}}$ siècle. Il s'agit d'une encyclopédie rédigée en prose, sous forme d'un dialogue qui met en scène le philosophe Timéo et son disciple, le prince Placides. L'œuvre est pertinente pour l'histoire du français savant du point de vue terminographique. 
" mater », qui est a dire mere... Ainsi pouvons-nous affirmer que l'auteur introduit en français une nomenclature savante, à savoir genitaires et petis tesmoings [testicules], preape [pénis ${ }^{22}$ ], vulve et marris [matrice]; cette nomenclature étant directement calquée sur les mots latins ${ }^{23}$. La volonté d'appuyer les termes par des explications étymologiques était une pratique courante des écrits scientifiques médiévaux qui remonte jusqu'à Isidore de Séville (VII siècle). L'auteur utilise également l'expression vergue (semblant d'usage neutre) et, quant au mot vit, il lui assigne l'usage "en commune langue». Il ne s'agit donc pas d'une expression 《 vulgaire » ou « malséante », mais d'une expression « non savante $»^{24}$.

Le troisième texte que nous allons mentionner brièvement est la traduction anonyme de la Chirurgia d'Henri de Mondeville. Traité savant écrit en latin par le médecin de Phillipe le Bel entre 1306 et 1320, la Chirurgia est une somme des pratiques chirurgicales médiévales. La traduction en français est contemporaine à l'original latin (le manuscrit date de 1314), qu'elle suit presque mot à mot. La première partie du texte est consacrée à l'anatomie du corps humain, la description des organes génitaux et excréteurs en fait naturellement partie. Pour nommer ces organes dont il décrit ensuite la structure, le traducteur utilise directement, sans glose ou commentaire métalinguistique, les mots vit, coille, coillons, con et $\mathrm{cul}^{25}$. La traduction est légèrement postérieure au traité Placides et Timeo, mais ne fait point usage des termes proposés dans ce texte.

Ces trois documents médiévaux attestent d'une manière convaincante que les mots vit, coille, coillons, con ou cul représentent des dénominations neutres utilisées par le peuple pour désigner les parties du corps pudiques ${ }^{26}$. En dehors du milieu courtois, dont la bienséance instigue de ne pas parler de ces $«$ affaires $»^{27}$, ces expressions n'ont rien de vulgaire et leur emploi dans le milieu urbain et populaire peut être qualifié de non-marqué. ${ }^{28}$

De ces réflexions, il s'ensuit une conclusion cruciale pour notre propos. Dans nos évaluations relatives à la grossièreté ou à la vulgarité, il faut en effet distinguer le thème (ou la «chose » si l'on veut) et les mots qu'on utilise pour en parler. Si la thématique sexuelle a été proscrite de l'éthique de la conversation courtoise, surtout pour les femmes, cela ne veut pas dire pour autant que les dénominations directes

\footnotetext{
${ }^{22}$ Dans le texte Placides et Timeo, le mot preape signifiait « pénis » tout court et non pas spécialement « pénis en érection », sens qu'on connaît aujourd'hui pour la forme priape.

${ }^{23}$ Dans les textes médiévaux, l'expression preape n'est pas attestée en dehors du traité Placides et Timeo. Quant à la forme priape, attestée dès 1515 ( $c f$. TLFi), elle fait fortune aujourd'hui dans le discours explicite obscène des sites pornographiques. Le calque petits tesmoins ne connaît guère un usage fréquent, vite évincé, dans le discours savant, par le latinisme testicules.

${ }^{24}$ Selon le TLFi, le mot vit provient du latin vectis «levier, barre».

${ }^{25}$ Édition de Dr. A. Bos, Tome I, pp. 123-128. Cf. p. ex. : 487 La partie de hors a .2. parties : c'est le vit et la coille. ; 496 Et a .2. pertuis : l'un est desus par lequel il pisse, l'autre est desous par lequel il met semence eu con. ; 516 Peri // toneon est continué de la part dedans o la coille duc' au trou du cul. Vit traduit le latin verga, coille traduit osseum, coillons traduit testiculi, le mot con n'a pas de correspondant lexical dans l'original latin.

${ }^{26}$ Notre analyse de ces trois textes confirme les observations que fait $\mathrm{P}$. Ménard à propos de l'usage des mots cul et merde (MENARD, 1983 :151) dans les romans courtois : ces mots relèvent du langage commun et n'ont pas de substitut savant dans la langue médiévale.

${ }^{27}$ C'est ainsi qu'il faut comprendre les vers 2109-2114 et 6898-6901 du Roman de la Rose.

${ }^{28}$ Cf. l'adjectif propre utilisé par Jean de Meun (v. 7103).
} 
(«propres ») des parties du corps pudiques soient elles-mêmes grossières, vulgaires, voire argotiques. Comme nous l'avons vu, le lexique médiéval ne disposait pas, jusqu'à Placides et Timeo du moins, de termes savants pour désigner ces organes. Comme toute valeur dans la langue est basée sur les oppositions, les statuts diastratiques, eux aussi oppositifs et différentiels, ne peuvent se constituer qu'à partir du moment où la langue a à sa disposition d'autres expressions auxquelles elle assigne, par l'intermédiaire d'une autorité énonciative (tel un clerc savant), un statut « haut ». Ainsi, le mot main est, quant à sa valeur diastratique, neutre, l'expression loche qui désigne la même partie du corps, est une variante familière (voire argotique selon DMF). Dans le même ordre d'idées, le mot vit est diastratiquement neutre, en revanche baudö̈n serait familier. Mais « parler de la main » ne représente aucune entrave à la bienséance courtoise, alors que « parler du vit » est difficilement tolérable. Cette tension qui existe entre les valeurs des mots et les valeurs comportementales donne lieu à de nombreuses situations comiques, que les fabliaux mettent en scène pour amuser les lecteurs.

\subsection{Normes comportementales et choix lexicaux - stratégies discursives des fabliaux}

\subsubsection{La bienséance linguistique}

La transgression de la norme conversationnelle courtoise, basée sur l'attitude réservée qu'éprouve la société «bien éduquée » à parler de la thématique sexuelle, pourtant alléchante tant pour les princesses que pour les bourgeoises ou les « vilaines », est très bien reflétée par les fabliaux. Elle est d'ailleurs très souvent au cœur même de l'effet comique du texte. Ainsi, le motif d'une jeune fille qui ne veut pas ou ne sait pas parler des affaires sexuelles exploite pleinement cette tension qui oppose le désir naturel et la bienséance sociétale. Les hommes séducteurs dont l'objectif principal est de faire l'amour avec une fille pudique inventent tout un discours figuré, parfois très ingénieux, pour parler de la «chose ». Naturellement, ils réussissent dans leur entreprise, car la pudicité de la fille n'est qu'un décor qui cache son désir insatiable, et la métaphore inventée sert de cadre pour la description de l'acte. À titre d'exemple, citons le fabliau De la Damoiselle qui n'ot parler de fotre qui n'aüst mal au cuer ( ${ }^{\circ} 111$, éd. M-R). Au fil de l'histoire, la demoiselle délicate se trouve toute seule avec un jeune séducteur qui se met à l'interroger sur les noms qu'elle donne aux parties sexuelles de son corps. Pour lui répondre, la fille déploie une métaphore filée dont le jeune homme sait bien profiter. Ainsi nommet-elle les poils pubiens «prez», le vagin "fontaine », l'anus est le «cornerres qui la garde » et qui se met à sonner lorsqu'une bête vient s'abreuver à la fontaine. Jouant le jeu, le jeune homme à son tour nomme son pénis «polains», les testicules sont deux «mareschaux». Comme on peut s'y attendre, la jeune fille invite le poulain à aller boire dans sa fontaine ; les deux « mareschaux » battrons le sonneur au cas où il se mettrait à sonner. Du point de vue discursif, les choix lexicaux relatifs à la dénomination des organes en question sont significatifs : dans le discours direct des deux protagonistes, les organes sont toujours nommés par une expression indirecte. Soit, ils les désignent par une expression de sens général : «Et que est ce 
en mi cest pré Ceste fosse soeve et plaine? " ou "Daviet, Que est or ce, en ce sachet? " Fait ele, "sont ce. II. luisiaus ? ", soit ils utilisent les dénominations métaphoriques décrites ci-dessus. Par contre, lorsque c'est le narrateur qui prend la parole, il utilise les expressions directes : Si trove la coille velue; Les. II. coillons taste et remue, ou, pour commenter l'invitation que fait la jeune fille au jeune homme à faire boire son poulain dans sa fontaine, le narrateur dit sans ambages : Atant li met el con lo vit. Aux jeux de mots déployés par les deux protagonistes, dont le but est de contourner les interdits d'une morale courtoise, le narrateur oppose une franchise de la parole qui contraste avec la bienséance linguistique de la société «bien éduquée ».

Un autre conte célèbre reflète clairement tous les aspects discursifs que nous venons de mentionner : le fabliau De l'Escuiruel (n¹21, éd. M-R). Dans ce texte, le narrateur met en scène une mère qui fait à sa fille une leçon de bonne conduite. Le comportement langagier fait naturellement partie des bonnes mœurs des milieux sociaux aisés: Quar a mal puet l'en atorner Fame quant l'en l'ot trop parler Autrement que ele ne doit. Dans ce sens, le péché le plus grave est de parler des organes sexuels de l'homme : Et une chose vous desfent Sor toutes autres mout trés bien, Que ja ne nommez cele rien Que cil homme portent pendant. Mais plus la mère insiste, plus la fille veut entendre le mot qui nomme cette «rien». Au bout de quelques tractations lors desquelles la fille avance tout un tas d'expressions en vue d'entendre la «bonne » (pesche, pendeloche, loche), la mère cède et dévoile à la fille le «nom » tant désiré : Je te di bien que ce est vit. À entendre ce mot, la fille exalte de joie et se met à répéter le mot, ce qui lui procure un plaisir malin : "Vit, » dist ele, "Dieu merci, vit! Vit dirai je, cui qu'il anuit, Vit, chetive! vit dist mon pere, Vit dist ma suer, vit dist mon frere, Et vit dist nostre chamberiere, Et vit avant et vit arriere Nomme chascuns à son voloir. Vous meïsme, mere, por voir, Dites vit, et je toute lasse Qu'ai forfet que vit ne nommaisse? Vit me doinst Dieus que je n'i faille! ». Un jeune homme a entendu cette conversation. Concluant que les paroles signalent le désir corporel qu'éprouve la fille, il vient auprès d'elle et il lui montre son pénis en prétendant que c'est un écureuil. Il évite volontairement le mot vit que pourtant la fille vient d'apprendre. Un détour linguistique qui fait atténuer, d'une manière hypocrite, le caractère immoral de l'acte. La fille joue le jeu, car l'écureuil, contrairement au vit, ne représente aucun danger pour son intégrité. Ainsi, les testicules deviennent deux œufs, l'écureuil, qui se nourrit des noix, est invité à aller en chercher dans le ventre de la jeune fille et il en mange autant qu'à la fin il vomit. La jeune fille prétend que le liquide vient de «l'œuf » brisé : «Tu as hurté de tel aïr [...] Que. I. des oes est esquaté [...] L'aubun m'en cort par mi les nages! ».

\subsubsection{L'usage des dénominations directes}

En dehors des cas où les auteurs thématisent cette bienséance discursive courtoise pour s'en moquer, les dénominations directes sont utilisées sans embarras, tant dans le discours du narrateur que dans le discours direct des protagonistes. Ainsi n'est-il pas rare que les femmes parlent de «mon con» : «Biaus amis, car metomes non $A$ vostre rien et à mon con. » (De porcelet, $\left.\mathrm{n}^{\circ} 101\right)$; "Sire, » fait ele, "ne vos hastez : Se vos volez voz volantez Faire de moi ne de mon con, I covient que 
beneïçon » (De l'evesque qui beneï lo con, $\mathrm{n}^{\circ}$ 77). La réplique suivante, mise dans la bouche d'un prêtre, est l'une des plus explicites De la Sorisete des estopes $\left(\mathrm{n}^{\circ} 105\right)$ : Je voil, «fait il, » vit avant traire: Si vos fotrai se j'onques puis, Se vostre con delivre truis.

Le fait que ces mots se trouvent dans un discours direct peut corroborer l'hypothèse selon laquelle ces expressions ont un statut neutre du point de vue diastratique $^{29}$. Nous nous gardons toutefois de surestimer ce critère, car il pourrait aussi être interprété d'une manière inverse. Il ne faut pas oublier en effet que les fabliaux sont d'abord des contes à rire. Dans le fabliau Du sot chevalier $\left(\mathrm{n}^{\circ} 20\right)$, nous assistons à une conversation entre un chevalier et une dame, conversation qui prend toutes les allures d'un dialogue courtois. Les deux locuteurs se vouvoient, ils s'appellent sire et dame, mais dans leurs répliques, ils utilisent sans ambages les mots vit et couille: Gardez là ne voist vostre vis, [...] Dame, dist-il, volez-vous donc Que mete mon vit ou plus lonc? [...] Et que ferai-je de ma coille? L'effet comique peut justement être recherché par le «conflit» des registres - les formules bienséantes de la conversation courtoise sont garnies de mots que la courtoisie cherche à éviter. Ce principe humoristique est sans doute à l'œuvre lorsque le narrateur décrit la rencontre entre le chevalier et la dame: Et puis a les jambes ouvertes, Se li monstra dant Connebert... Comme nous l'avons montré ci-dessus : si les mots eux-mêmes sont certes « neutres », la matière, en revanche, ne l'est pas.

Dans d'autres cas, l'effet comique est atteint par une répétition excessive des mots qui désignent les organes sexuels. Le genre fabliesque licencie l'usage de ces expressions, c'est d'ailleurs l'une des marques les plus saillantes du genre, il n'est donc pas étonnant que les auteurs en profitent à foison. $C f$. les passages suivantes (Les .IIII. Souhais saint Martin, $\mathrm{n}^{\circ}$ 133) : Adonc fu ele bien connue Qu'ele ot. II. cons en la veûe, IIII. en ot ou front coste à coste, Et con devant et con d'encoste; Si ot con de mainte maniere Et con devant et con derriere, Con tort, con droit et con chenu, Et con sanz poil et con velu, Et con pucel, et con estrait, Et con estroit, et con bien fait, Et con petit, et con aorce, Et con parfont et con seur boce, Et con au chief, et con aus piez, Adonques fu li vilains liez... Et le même motif du côté masculin : Quant ele ot souhaidié et dit, Au vilain saillirent li vit; Li vit li saillent par le nez Et par la bouche de delez; Si ot vit lonc et vit quarré, Vit gros, vit cort, vit reboulé, Vit corbe, vit agu, vit gros; Sor le vilain n'ot si dur os Dont vit ne saillent merveillous. Li vit li saillent des genous; Por Dieu or entendez merveilles, Li vit li saillent des oreilles, Et par devant en contremont Li sailli uns grans vis du front, Et par aval dusques aus piez Fu li vilains de vis chargiez...

Conséquemment, les auteurs se sentent libres de décrire ces organes à loisir. Les substantifs en question se voient apposer des adjectifs qui en désignent les qualités ${ }^{30}$. Ainsi, le vit est typiquement gros, agu, corbe, grant, quarré, long, noir, roides, fier ; le con est douce, chenu, velu, grant; les coilles sont granz, grosses, velues, merveilleuses, noires. Dans cette optique, le fabliau Fevre de Creel $\left(\mathrm{n}^{\circ} 21\right)$ se

\footnotetext{
${ }^{29}$ C'est ce que suggère P. NYKROG $(1973: 211)$ sur la base de ce critère.

${ }^{30}$ L'une des fonctionnalités des corpus TXM permet de calculer les co-occurrences les plus fréquentes d'un mot-vedette.
} 
distingue particulièrement - il contient une description très explicite du sexe masculin qui s'étale sur une trentaine de vers.

\subsubsection{Expressions métaphoriques}

Plus haut (section 3), nous avons soulevé la question relative au statut des expressions métaphoriques. Nous avons fait observer également que parmi les entrées du DMF, les éléments métaphoriques sont absolument majoritaires. L'analyse des fabliaux nous permet de compléter et de nuancer ces observations.

Dans la langue des fabliaux, deux cas de figure sont à distinguer: 1) des expressions qui fonctionnent comme dénomination de ces organes uniquement au sein d'un seul texte, typiquement dans le cadre d'une métaphore filée ; et 2) des expressions qui sont utilisées en dehors des contextes métaphoriques complexes et que l'on trouve même dans plusieurs textes à la fois. Les premières n'ont qu'un statut lexical éphémère et on aurait tort de les inclure sur les listes lexicographiques de portée générale. Citons à titre d'exemple: escuiruel, polains, connetiaus, forment (pénis) ; fontaine, porcelet (vagin) ; luisaus, maréchaus, oes, roignons (testicules). Parmi les secondes on peut citer : baudoïn, andoille, pendeloche, rien, ostil, boiel (pénis); traus, Golyas (vagin). Ces expressions devaient avoir une certaine stabilité lexicale, car dans les textes, elles sont employées directement, sans glose, et ne font partie d'aucune série métaphorique filée (p. ex. " animalière » ou « nutritionnelle »). Dans une perspective lexicographique, leur présence éventuelle sur les listes des entrées serait ainsi justifiée. Remarquons toutefois que les expressions comme rien, trou ou ostil sont à considérer plutôt comme des dénominations du type hypéronymique. Du point de vue fréquentiel, ces expressions indirectes sont assez marginales dans le corpus des fabliaux ${ }^{31}$, ce qui contraste d'une manière significative avec les fréquences des expressions directes ( $c f$. plus haut). Cette proportion lexicale est tout à fait conforme à la poétique des fabliaux : le francparler concourt pleinement aux effets comiques de ces contes à rire.

\section{Conclusion}

Notre enquête computationnelle a démontré, d'une manière objective et exacte, les spécificités lexicales des fabliaux dans le domaine des noms désignant les parties du corps. Nous avons vu que l'analyse de ces expressions, de leurs fréquences, de leur nature onomasiologique et de leur usage doit nécessairement tenir compte des facteurs culturels, sociaux et esthétiques. C'est uniquement dans cette perspective philologique, qui fait partie intégrante du travail du linguiste, que nous pouvons déterminer la valeur diastratique des expressions relevées dans le corpus. Le choix des mots qui nomment les parties du corps pudiques fait partie intégrante de la poétique des fabliaux, dont le but principal est de faire rire le public. Les stratégies discursives qui servent à mettre en relief les expressions obscènes ou, au contraire,

\footnotetext{
${ }^{31}$ P. ex. : baudoï $(\mathrm{FA}=1)$, andouille $(\mathrm{FA}=3)$, pendeloche $(\mathrm{FA}=2)$.
} 
à les inhiber reflètent les normes et les habitudes de la société médiévale si bien que l'étude lexicale devient inévitablement une étude sociolinguistique.

\section{BIBLIOGRAPHIE}

BÉDIER Joseph (1893), Les fabliaux : études de littérature populaire et d'histoire littéraire du Moyen âge, Paris, Bibliothèque de l'École des hautes études.

BFM - Base de Français Médiéval [en ligne]. Lyon: ENS de Lyon, Laboratoire IHRIM, 2019, <txm.bfm-corpus.org>.

Bloch R. Howard, (1993), Postface, in : Rossi Luciano, Straub Richard (éd.), Fabliaux érotiques, Paris, Librairie générale française, p. 531-545.

Boutet Dominique (1985), Les fabliaux, Paris, Presses Universitaires de France.

CORBELlARI Alain (2014), D'un recueil « complet » à l'autre Les répertoires de fabliaux, de Montaiglon-Raynaud au NRCF, in : COLLET Olivier, MAILlET Fanny, TRACHSLER Richard (éd.), L'Étude des fabliaux après le Nouveau recueil complet des fabliaux,), Paris, Classiques Garnier, p. 15-37.

DMF : Dictionnaire du Moyen Français, version 2015 (DMF 2015). ATILF - CNRS \& Université de Lorraine. Site internet : http://www.atilf.fr/dmf.

MÉnARD Philippe, (1983) Les fabliaux. Contes à rire du Moyen Âge, Paris, Presses Universitaires de France.

NYKROG Per (1973), Les Fabliaux : Nouvelle Édition, Genève, Librarie Droz.

PEŠEK Ondřej (2019), Korpusy a významové struktury: komputační sonda do textů moderního českého jazykovědného myšlení, in : Vladimír Papoušek et al. (éd.), Pokušeni neviditelného - Myšleni moderny, Praha, Akropolis, p. 144-181.

RASTIER François (2011), La mesure et le grain: sémantique de corpus. Paris, Honoré Champion éditeur.

ZINK Michel (1993), Avertissement, in : Rossi Luciano, STRAUB Richard (éd.), Fabliaux érotiques, Paris, Librairie générale française, p. 7-8.

\section{ÉDITIONS}

Recueil général et complet des fabliaux des XIIIe et XIVe siècles imprimés ou inédits, (1872), Montaiglon Anatole de, Gaston Raynaud (éd.), Paris, Librairie des bibliophiles.

DE LORRIS Guillaume, DE MEUN Jean, Roman de la Rose (1966), LECOY Félix (éd.). Paris, Champion, DE MEUN

DE LORRIS Guillaume, DE MEUN Jean (1992), Le Roman de la Rose. Édition d'après les manuscrits BN 12786 et BN 378, STRUBEL Armand (éd.), Paris, Librairie générale française.

Placides et Timéo ou Li secrés as philosophes (1980), THOMASSET C.-A. (éd.), Paris et Genève, Librairie Droz. 
La chirurgie de maître Henri de Mondeville, traduction contemporaine de l'auteur, publiée d'après le manuscrit unique de la Bibliothèque nationale par Dr A. Bos, (1897-1898) Paris, Firmin Didot (Société des anciens textes français). 